\title{
FLLL32, a curcumin analog, ameliorates intestinal injury in necrotizing enterocolitis
}

\author{
Jeffrey Eckert ${ }^{\prime}$ \\ Brian Scott ${ }^{1,2}$ \\ Shelley M Lawrence ${ }^{3}$ \\ Michael Ihnat ${ }^{4}$ \\ Hala Chaaban' \\ 'Department of Pediatrics, University \\ of Oklahoma Health Sciences Center, \\ Oklahoma City, OK, ${ }^{2}$ Department \\ of Pediatrics, University of Texas \\ Health Science Center at San Antonio, \\ San Antonio, TX, ${ }^{3}$ Department of \\ Pediatrics, University of California San \\ Diego, San Diego, CA, ${ }^{4}$ Department of \\ Pharmaceutical Sciences, University \\ of Oklahoma, College of Pharmacy, \\ Oklahoma City, OK, USA
}

Correspondence: Hala Chaaban Division of Neonatal-Perinatal Medicine, OU Children's Hospital, 1200 Everett Drive, ETNP 7504, Oklahoma City, OK 73104, USA

Tel + I $40527 \mid 4755$

Fax + I 405 27I I236

Email hala-chaaban@ouhsc.edu
This article was published in the following Dove Press journal:

Journal of Inflammation Research

14 June 2017

Number of times this article has been viewed

Background: Necrotizing enterocolitis (NEC) is a devastating gastrointestinal disease that primarily affects premature infants. It is characterized by inflammation and leukocyte infiltration that can progress to intestinal necrosis, perforation, systemic inflammatory response, and death. In this study, we examined the effect of FLLL32, a curcumin analog, on an NEC-like neonatal intestinal injury model.

Methods: NEC was induced in CD-1 mice pups using the Paneth cell ablation and Klebsiella infection model. Pups were divided into sham, NEC, and NEC + FLLL32 groups. At the end of the experiment, pups were euthanized; whole blood and small intestines were harvested. Intestinal inflammatory cytokine profile, in vivo intestinal permeability using serum fluorescein isothiocyanate-dextran, and histological injury scores were compared between the groups.

Results and conclusion: FLLL32-treated pups had lower intestinal injury, improved intestinal permeability, and lower proinflammatory cytokine profiles compared to those in untreated pups with NEC. These results suggest that FLLL32 plays a protective role in NEC.

Keywords: necrotizing enterocolitis, neonatal intestinal inflammation, curcumin, FLLL32, STAT3 inhibitors

\section{Introduction}

Necrotizing enterocolitis (NEC) is a devastating gastrointestinal disease characterized by inflammation and injury to the small intestine. ${ }^{1,2}$ Despite advances in care, NEC remains the leading cause of morbidity and mortality in infants born weighing less than 1500 g. ${ }^{1,3,4}$ Although the etiology of NEC is unclear, evidence suggests that inflammation plays a central part in the pathogenesis. ${ }^{5,6}$ Specifically, activation of Toll-like receptor 4 (TLR4) is essential for the development of NEC. ${ }^{7-9}$ Thus, strategies to inhibit TLR activation or inflammatory cytokines have been proposed to help ameliorate injury in models of NEC. ${ }^{10,11}$

Curcumin is a natural compound found in Curcuma longa. ${ }^{12}$ Curcumin interacts with numerous targets that are involved in inflammation. ${ }^{12-15}$ It downregulates inflammatory cytokines, such as tumor necrosis factor-alpha (TNF- $\alpha$ ) and interleukin (IL)-1,

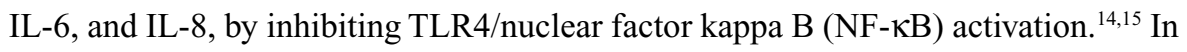
intestinal inflammation models, curcumin attenuates inflammation and injury through inhibition of COX-2 expression ${ }^{16}$ and the Janus kinase (JAK)/signal transducers and activators of transcription (STAT) signaling pathways. ${ }^{17-20}$

The safety and tolerability of curcumin at high doses are well established. However, under physiologic conditions, curcumin is unstable and has poor bioavailability. FLLL32 is a diketone analog of curcumin that exhibits improved potency and biochemical 
properties, specifically as an inhibitor of the inflammatory signaling molecule Jak2/STAT3. ${ }^{21,22}$ The use of FLLL32 in intestinal inflammation has not been explored. Thus, the purpose of this study was to determine the effect of FLLL32 in a neonatal NEC-like intestinal inflammation model.

\section{Methods}

\section{Effect of FLLL32 and curcumin on IL- 6-induced reduction of transepithelial electrical resistance (TEER) in T84 monolayer}

T84 intestinal epithelial cells (CCL-248) purchased from the American Type Cell Culture (ATCC, Manassas, VA, USA) were grown under standard conditions as described previously. ${ }^{23,24}$ Cells were seeded in 24-well Transwells (Cat\#: 353495 , $6.5 \mathrm{~mm}$ diameter, $0.4 \mathrm{~mm}$ pore size, PET track-etched membrane) at a density of $6 \times 10^{4}$ cells $/ 800 \mathrm{~mL} /$ well (BD Biosciences, San Jose, CA, USA). Cells were grown for 7 days on the Transwells prior to the experiments, until TEER indicated established tight junction formation (TEER $>500 \Omega \mathrm{cm}^{2}$ ). One day prior to the experiment, cells were serum starved overnight. Cells were pretreated with FLLL32 $(50 \mu \mathrm{M})$, curcumin $(50 \mu \mathrm{M})$, or carrier (dimethyl sulfoxide) for 1 hour in serum-free medium. Recombinant human IL-6 (10 ng/mL) was applied to the cell monolayers (Miltenyioe Biotech, San Diego, CA, USA). After 60 minutes, the cells were supplemented with serum, and TEER, as an indicator of tight junction permeability, was measured at 24, 48, and 72 hours. ${ }^{24}$ TEER was measured using Millcell-ERS (Millipore, Bedford, MA, USA) and calculated as ohms $\times \mathrm{cm}^{2}$ $\left(\Omega \mathrm{cm}^{2}\right)$ by multiplying it by the surface area of the monolayer. Changes in TEER during experimental conditions were calculated as the percentage of corresponding basal values. Triplet cell monolayers were used for each group in each experiment.

\section{Determination of no-observable-adverse- effect-level (NOAEL)}

To determine the safety and tolerability of FLLL32 (Calbiochem $\left.{ }^{\circledR}\right)$ in neonatal mice, P8-P14 CD-1 mouse pups were orally gavaged 25 and $50 \mathrm{mg} / \mathrm{kg}$ of FLLL32 at a volume of $10 \mu \mathrm{L} / \mathrm{g}$ once daily at the same time every day for 7 days. The pups were weighed daily and observed for any adverse effects, such as lethargy, abnormal behavior, weight loss, and death.

\section{Experimental model of NEC}

All animal procedures were approved by the Institutional Animal Care and Use Committee at the University of Oklahoma Health Sciences Center and were performed in strict accordance with the recommendations in the Guide for the Care and Use of Laboratory Animals. Briefly, CD-1 (Charles River Laboratory) pups were divided into three groups: 1) Sham (n=6), 2) NEC $(n=18)$, and 3) NEC + FLLL32 $(n=12)$. The NEC + FLLL32 group received $25 \mathrm{mg} / \mathrm{kg}$ of FLLL32 by oral gavage from P9 to P14. This was based on prior studies and the NOAEL effects listed previously. ${ }^{22}$ NEC was induced at P15 using the Panethcell ablation and Klebsiella infection model, also known as the dithizone/Klebsiella model (DK model). ${ }^{25}$ Pups received an intraperitoneal (ip) injection of $33 \mathrm{mg} / \mathrm{kg}$ of dithizone (Sigma-Aldrich, St. Louis, MO, USA) or an equivalent volume of vehicle alone at P15. Six hours after the injection, mice pups received an enteral gavage of $10^{8} \mathrm{CFU} / \mathrm{g}$ body weight Klebsiella pneumoniae (ATCC\#10031, Manassas, VA, USA). Mice pups were then continuously monitored for 10 hours after which they were euthanized, and blood and tissues were collected.

\section{Histological evaluation of NEC}

Tissues were fixed with $10 \%$ formalin buffer and stained with hematoxylin and eosin for microscopic examination. Mucosal injury was evaluated by two blinded pathologists and graded on a 4-point scale: grade 0, no injury (normal); grade 1, separation of the villous core; grade 2, villous core separation and epithelial sloughing; and grade 3 , denudation of epithelium with loss of villi, full-thickness necrosis. The analysis was performed on three to five sections. Animals with histological scores $\geq 2$ were defined as having NEC. ${ }^{26}$

\section{In vivo intestinal barrier function assay}

To investigate the effect of FLLL32 on intestinal permeability barrier function in the NEC model, an in vivo permeability assay was performed using fluorescein isothiocyanate (FITC)-dextran. ${ }^{27,28}$ Surviving mice pups were gavaged $4 \mathrm{kDa}$ FITC-dextran (44 mg/100 g body weight). After 4 hours, blood was collected and centrifuged at $4^{\circ} \mathrm{C}, 3000 \mathrm{~g}$ for $10 \mathrm{~min}$. Plasma was diluted in an equal volume of phosphatebuffered saline (PBS) and analyzed for DX-4000-FITC concentration with a fluorescence spectrophotometer (Tecan, Maennedorf, Switzerland) at an excitation wavelength of $480 \mathrm{~nm}$ and emission wavelength of $520 \mathrm{~nm}$.

\section{Mouse inflammatory cytokine quantification}

Small intestinal samples were homogenized in lysis buffer. Intestinal cytokines were analyzed using custom designed Procarta Plex Mouse Cytokine \& Chemokine Panel 1A (eBioscience, San Diego, CA, USA) as per manufacturer instructions. Final cytokine levels were normalized to total protein concentration (milligram/milliliter) and reported as pictogram/microgram protein. 


\section{Statistical analysis}

Data are presented as mean \pm standard error of mean (SEM) and analyzed using one-way analysis of variance (ANOVA) followed by Dunnett's multiple comparisons test. Data were considered significant when $p<0.05$. All the analyses were performed using GraphPad Prism version 6.00 for Windows (GraphPad Software, La Jolla, CA, USA).

\section{Results}

\section{FLLL32 protects T84 monolayers against inflammation-induced paracellular permeability}

To evaluate the effect of FLLL32 on intestinal permeability in vitro, we measured the TEER in T84 cell monolayers. Similar to previous studies, ${ }^{24}$ IL-6 decreased the TEER in a time-dependent manner, indicating increased tight junction permeability to ionic solutes. As seen in Figure 1, the addition of $10 \mathrm{mg} / \mathrm{mL}$ IL- 6 to the culture medium for 72 hours induced more than $25 \%$ decrease in the TEER reading compared to baseline in a time-dependent manner. Pretreatment with FLLL32 ameliorated IL-6 effect on TEER at 24, 48, and 72 hours (Figure 1). In contrast to curcumin pretreatment, at the same molar concentration, FLLL32 had no effect on IL6-induced reduction in TEER at 24 and 48 hours. At 72 hours, a modest alleviation on TEER is seen when compared with FLLL32 pretreatment. These results could suggest that
FLLL32 is more superior to curcumin in preserving barrier function in vitro compared to curcumin.

\section{Determination of NOAEL}

Although the use of FLLL32 has been used extensively in different murine models of malignancy, its use in neonatal mice pups has not been reported. Thus, we sought to determine its safety and tolerability in CD-1 mice pups for 7 days starting P8 till P 14 . We administered FLLL32 orally at two different doses, $25 \mathrm{mg} / \mathrm{kg}$ and orally $50 \mathrm{mg} / \mathrm{kg}$ at a volume of $10 \mu \mathrm{L} / \mathrm{g}$ once daily. The pups were weighed daily and observed for any clinical signs of distress such as lethargy, abnormal behavior, weight loss, or death. Pups receiving $25 \mathrm{mg} / \mathrm{kg}$ of FLLL32 had no clinical signs of adverse events and had no difference in weight gain compared to controls. In contrast, pups receiving $50 \mathrm{mg} / \mathrm{kg}$ daily had lower weight gain on days 6 and 7 compared to both control and the $25 \mathrm{mg} /$ kg group (Figure 2).

\section{FLLL32 reduces incidence and severity of intestinal injury in DK NEC model}

NEC was induced using the DK NEC model as described in the "Methods" section. Figure 3A-C shows representative histological pictures of sections obtained from the three groups. As expected, sham pups had normal villi and submucosal structure (Figure 3A). The untreated NEC group

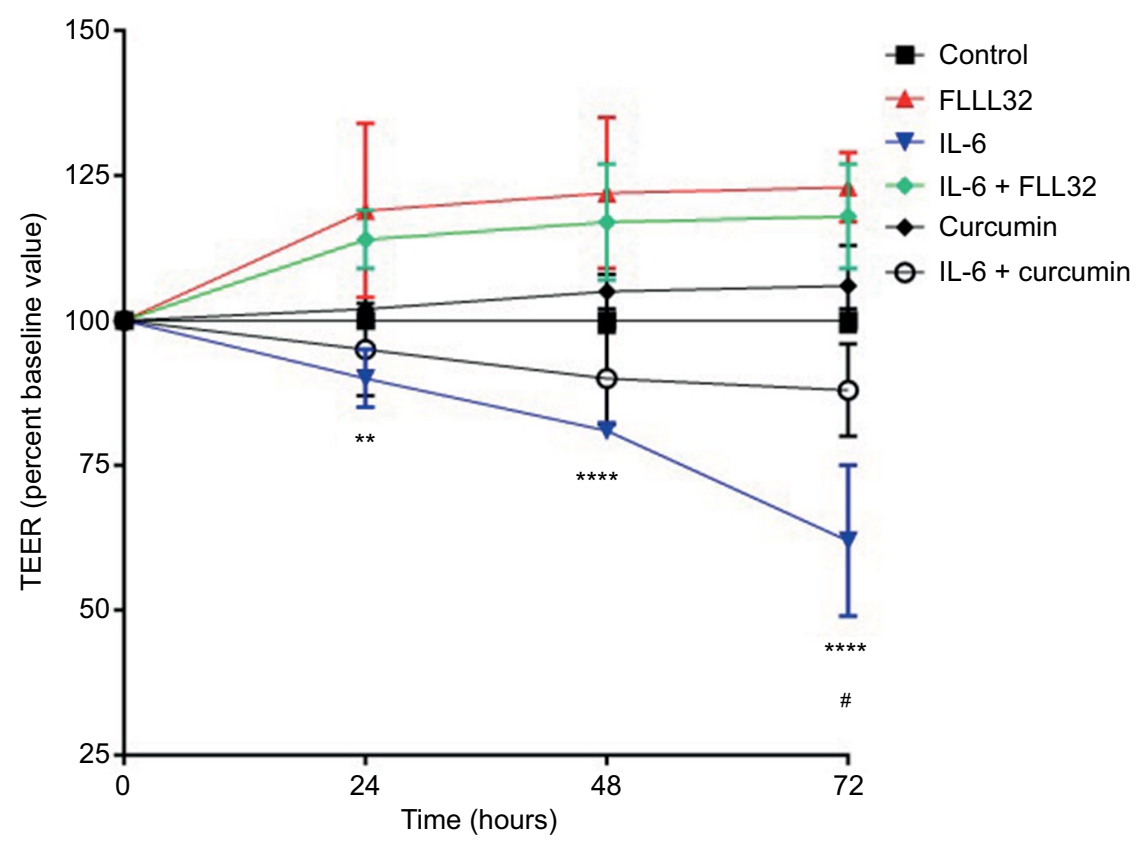

Figure I Effect of FLLL32 and curcumin on IL-6-induced reduction of TEER in T84 monolayer.

Notes: TEER value of T84 monolayers incubated with cell culture medium for $0-72$ hours in the presence of IL-6 (I0 ng/mL) with FLLL32 (50 $\mu$ M), curcumin ( $50 \mu M)$, or carrier (dimethyl sulfoxide) for I hour in serum-free medium. ${ }^{* *} p=0.00$ I (IL-6 vs. IL-6 + FLLL32 at 24 hours), $* * * * p<0.000$ I (IL-6 vs. IL-6 + FLLL32 at 48 and 72 hours), and \# $p=0.003$ (IL-6 vs. IL-6 + curcumin).

Abbreviations: IL, interleukin; TEER, transepithelial electrical resistance. 


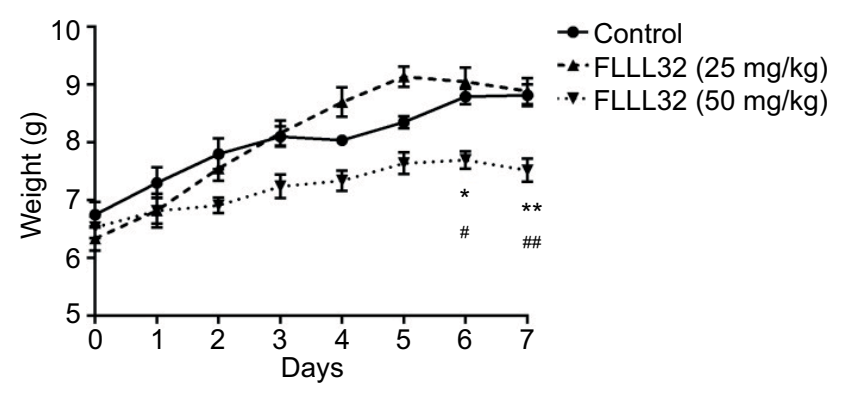

Figure 2 Determination of no-observable-adverse-effect-level (NOAEL). Notes: P8-PI4 CD-I mouse pups were orally gavaged 25 and $50 \mathrm{mg} / \mathrm{kg}$ of FLLL32 once daily for 7 days. Pups were weighed daily and observed for side effects. ${ }^{*} p<0.05$ (FLLL32 [50 mg/kg] vs. control at 6 days), ${ }^{* *} p<0.01$ (FLLL32 [50 mg/kg] vs. control at 7 days). ${ }^{\#} p<0.001$ (FLLL32 [50 mg/kg] vs. FLLL32 [25 mg/kg] at 6 days). ${ }^{\#} p<0.000$ I (FLLL32 [50 mg/kg] vs. FLLL32 [25 mg/kg]).

had moderate to severe intestinal villi damage (Figure 3B). More importantly, pups in the NEC + FLLL32 had mild evidence of intestinal injury (Figure 3C). To further determine the incidence and severity of NEC in the groups, we used the modified NEC scoring system. ${ }^{26}$ Pretreatment with FLLL32 reduced intestinal injury score from a mean of 2 in the untreated group to a mean of 0.33 in the NEC + FLLL32 group (Figure 3D). In addition, the FLLL32 reduced the incidence of NEC from $72 \%(13 / 18)$ in the untreated NEC group to $8 \%(1 / 12)$ in the treated group $(p=0.001)$.

\section{FLLL32 protects intestinal barrier in a DK NEC model}

Although the etiology of NEC remains incompletely understood, evidence shows that breakdown of the intestinal barrier is a predisposing factor in the development of NEC. ${ }^{9,29-31}$ To determine whether FLLL32 protection against NEC was associated with preservation of intestinal barrier, intestinal permeability was assessed by oral administration of the FITC-dextran ( $4 \mathrm{kDa})$ to all surviving pups. Concentrations of FITC-dextran in serum were measured 4 hours after administration. As shown in Figure 3E, the sham group had low FITC-dextran levels in the blood. Pups in the untreated NEC group had the highest levels of FITC-dextran in the blood, reflecting increased intestinal permeability. Pups in $\mathrm{NEC}+$ FLLL32 group had significantly decreased FITCdextran in blood to near sham levels, indicating that pretreatment with FLLL32 protected intestinal barrier $(p<0.0001)$.

\section{FLLL32 decreases intestinal inflammatory cytokine protein levels in DK NEC model}

Consistent evidence demonstrates the important role of inflammatory cytokines in the pathogenesis of NEC. ${ }^{5-9,32}$ To determine the effect of FLLL32 on intestinal cytokine levels, we compared the levels of IL-1 $\beta$, IL-6, growthregulated oncogene-alpha (GRO- $\alpha)$, and TNF- $\alpha$ in intesti- nal tissue lysates from pups in the three groups. Similar to other studies, ${ }^{25}$ levels of all inflammatory cytokines were significantly increased compared to sham (Figure 3F-I). Interestingly, pups in the NEC + FLLL32 group resulted in 2.5-fold decrease in TNF- $\alpha$ (Figure 3F, $p=0.001$ ), 5.8-fold decrease in IL-6 (Figure 3G, $p<0.001$ ), 4.8-fold decrease in IL-1 $\beta$ (Figure 3H, $p=0.009$ ), and 5-fold decrease in GRO- $\alpha$ levels (Figure 3I, $p=0.034$ ) compared to pups in the untreated NEC group. Intestinal IL-10 levels did not differ between NEC + FLLL32 and untreated NEC groups (data not shown).

\section{Discussion}

NEC is a devastating disease characterized by inflammation and injury to the small intestine. Despite early and aggressive treatment, NEC can quickly progress to frank bowel necrosis and death. ${ }^{4,33}$ Although the pathogenesis of NEC is still unclear, observations suggest a complex interplay between prematurity, formula feeding, hypoxia, and altered bacterial colonization..$^{9,34,35}$ In premature infants, developmental immaturity of mucosal barrier and increased expression of TLR4 on the surface of the intestinal epithelium render the gut highly reactive and susceptible to injury from various stimuli. ${ }^{7}$ This leads to an exaggerated proinflammatory cytokine release, leukocyte infiltration, altered epithelial barrier, intestinal necrosis, and bacterial translocation across the lumen. ${ }^{36,37}$

FLLL32 is a curcumin analog with improved solubility and bioavailability compared to the parent product. $^{22}$ Although FLLL32 has been extensively used in cancer models, ${ }^{22,38,39}$ its use in inflammation, specifically in NEC, has not been explored. Our results demonstrate that FLLL32 given orally at $25 \mathrm{mg} / \mathrm{kg}$ is well tolerated in neonatal mice pups. We also show that similar to curcumin, ${ }^{16}$ prophylactic administration of FLLL32 reduces the incidence and severity of NEC in DK mode. FLLL32 also preserved intestinal permeability and reduced intestinal proinflammatory cytokines, both of which play important roles in the pathogenesis of NEC. ${ }^{5,6,9,29-31}$ Although we did not perform a comparison of FLLL32 and curcumin in NEC, our results show that FLLL32 was superior to curcumin in preserving intestinal barrier function in vitro. The protective mechanism of FLLL32 could be through STAT3 inhibition, which has been shown to modulate PAF-induced TLR4 expression in intestinal epithelial cells in vitro. ${ }^{40}$

\section{Conclusion}

Oral FLLL32 decreases intestinal inflammation and injury in a DK NEC model. Further studies are needed to better understand the mechanisms underlying its protective effects. 


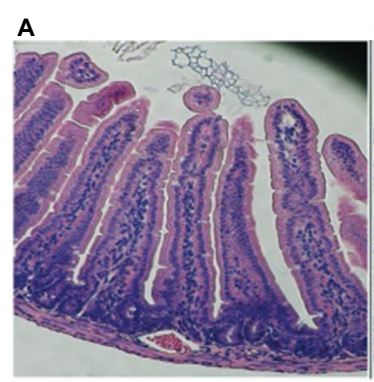

B

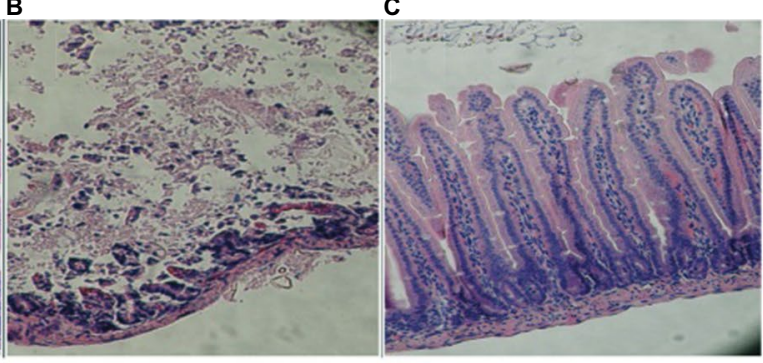

D

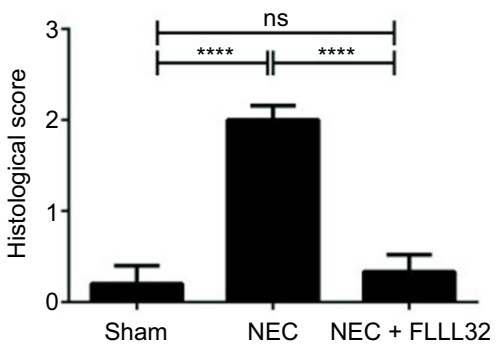

$\mathbf{F}$

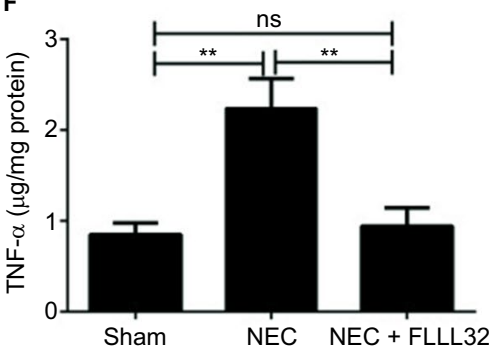

H

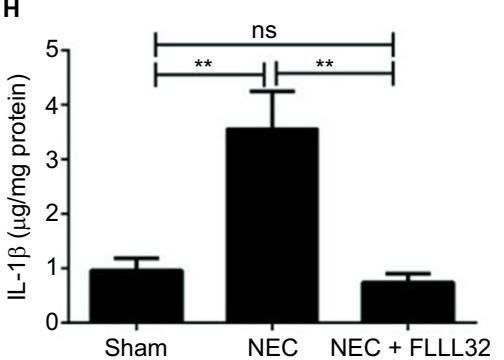

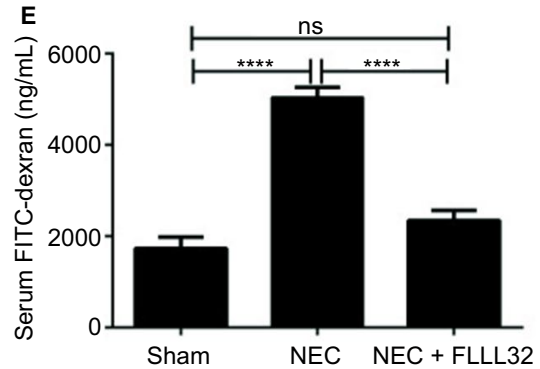

G
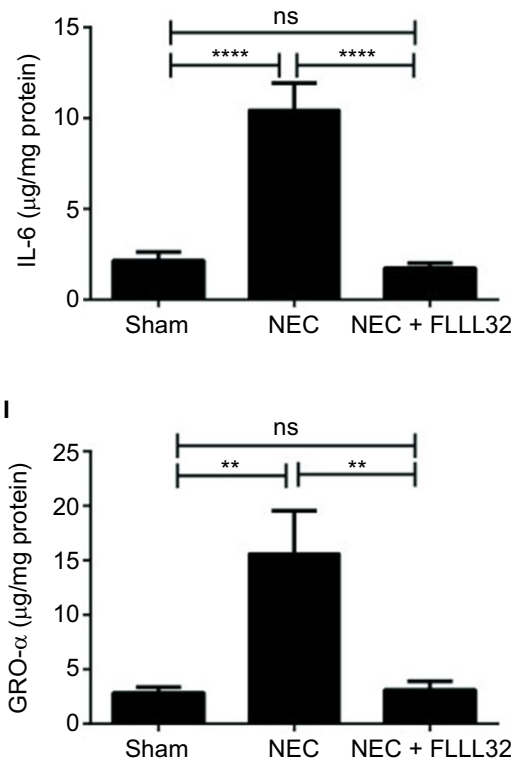

Figure 3 FLLL32 attenuates intestinal inflammation and injury in DK NEC model.

Notes: Representative H\&E pictures from pups in the sham group (A), untreated NEC group (B), and NEC + FLLL32 group (C); 20× magnification. (D) Histological NEC scoring was obtained by two pathologists blinded to the groups. (E) FLLL32 preserved intestinal permeability in the NEC + FLLL32 group compared to the untreated group and control group. FLLL32 pretreatment reduced the levels of proinflammatory cytokines, TNF- $\alpha(\mathbf{F}, p=0.00 \mathrm{I}), \mathrm{IL}-6$ (G, $p<0.00 \mathrm{I})$, IL-I $\beta$ (H, $p=0.009)$, and GRO- $\alpha$ levels $(\mathbf{I}, p=0.034)$ compared to pups in the untreated NEC group. Data are mean \pm SEM. Results are representative of at least three separate experiments. $* * p<0.05$; $* * * * p<0.00$ I. Abbreviations: DK, dithizone/Klebsiella; FITC, fluorescein isothiocyanate; GRO- $\alpha$, growth-regulated oncogene-alpha; H\&E, hematoxylin and eosin; NEC, necrotizing enterocolitis; TNF- $\alpha$, tumor necrosis factor-alpha; IL, interleukin; SEM, standard error of mean; ns, not significant.

\section{Acknowledgments}

We would like to acknowledge Randle Gallucci, PhD, and Kathryn Burge, $\mathrm{PhD}$, in helping with manuscript editing. This paper was presented in part at the Pediatric Academic Society Meeting, April 28, 2015.41

\section{Disclosure}

The authors report no conflicts of interest in this work.

\section{References}

1. Neu J, Walker WA. Necrotizing enterocolitis. $N$ Engl $J$ Med. 2011;364(3):255-264.

2. Rowe MI, Reblock KK, Kurkchubasche AG, Healey PJ. Necrotizing enterocolitis in the extremely low birth weight infant. J Pediatr Surg. 1994;29(8):987-990. discussion 990-981.

3. Patel RM, Kandefer S, Walsh MC, et al; Eunice Kennedy Shriver National Institute of Child Health and Human Development Neonatal Research Network. Causes and timing of death in extremely premature infants from 2000 through 2011. N Engl J Med. 2015;372(4):331-340. 
4. Srinivasan PS, Brandler MD, D’Souza A. Necrotizing enterocolitis. Clin Perinatol. 2008;35(1):251-272, x.

5. Frost BL, Jilling T, Caplan MS. The importance of pro-inflammatory signaling in neonatal NEC. Semin Perinatol. 2008;32(2):100-106.

6. Caplan MS, Simon D, Jilling T. The role of PAF, TLR, and the inflammatory response in neonatal necrotizing enterocolitis. Semin Pediatr Surg. 2005;14(3):145-151.

7. Sodhi CP, Shi XH, Richardson WM, et al. Toll-like receptor-4 inhibits enterocyte proliferation via impaired beta-catenin signaling in necrotizing enterocolitis. Gastroenterology. 2010;138(1):185-196.

8. Egan CE, Sodhi CP, Good M, et al. Toll-like receptor 4-mediated lymphocyte influx induces neonatal necrotizing enterocolitis. $J$ Clin Invest. 2016;126(2):495-508.

9. Leaphart CL, Cavallo J, Gribar SC, et al. A critical role for TLR4 in the pathogenesis of necrotizing enterocolitis by modulating intestinal injury and repair. J Immunol. 2007;179(7):4808-4820.

10. Neal MD, Jia H, Eyer B, et al. Discovery and validation of a new class of small molecule Toll-like receptor 4 (TLR4) inhibitors. PLoS One. 2013;8(6):e65779.

11. Good M, Siggers RH, Sodhi CP, et al. Amniotic fluid inhibits Toll-like receptor 4 signaling in the fetal and neonatal intestinal epithelium. Proc Natl Acad Sci U S A. 2012;109(28):11330-11335.

12. Jurenka JS. Anti-inflammatory properties of curcumin, a major constituent of Curcuma longa: a review of preclinical and clinical research. Altern Med Rev. 2009;14(2):141-153.

13. Goel A, Kunnumakkara AB, Aggarwal BB. Curcumin as "Curecumin": from kitchen to clinic. Biochem Pharmacol. 2008;75(4):787-809.

14. Basnet P, Skalko-Basnet N. Curcumin: an anti-inflammatory molecule from a curry spice on the path to cancer treatment. Molecules. 2011;16(6):4567.

15. Zhong K. Curcumin mediates a protective effect via TLR-4/NF- $\kappa B$ signaling pathway in rat model of severe acute pancreatitis. Cell Biochem Biophys. 2015;73(1):175-180.

16. Jia SH, Wei H, Yu JL, Wei XD, Zhang XP, Li JC. [Protective effects of curcumin on neonatal rats with necrotizing enterocolitis]. Zhongguo Dang Dai Er Ke Za Zhi [Chin J Contemp Pediatr]. 2010;12(2):132-136.

17. Salh B, Assi K, Templeman V, et al. Curcumin attenuates DNBinduced murine colitis. Am J Physiol Gastrointest Liver Physiol. 2003;285(1):G235-G243.

18. Zhang X, Wu J, Ye B, Wang Q, Xie X, Shen H. Protective effect of curcumin on TNBS-induced intestinal inflammation is mediated through the JAK/STAT pathway. BMC Complement Altern Med. 2016;16(1):299.

19. Ali T, Shakir F, Morton J. Curcumin and inflammatory bowel disease: biological mechanisms and clinical implication. Digestion. 2012;85(4):249-255.

20. Lubbad A, Oriowo MA, Khan I. Curcumin attenuates inflammation through inhibition of TLR-4 receptor in experimental colitis. Mol Cell Biochem. 2009;322(1-2):127-135.

21. Liu Y, Fuchs J, Li C, Lin J. IL-6, a risk factor for hepatocellular carcinoma: FLLL32 inhibits IL-6-induced STAT3 phosphorylation in human hepatocellular cancer cells. Cell Cycle. 2010;9(17):3423-3427.

22. Bill MA, Nicholas C, Mace TA, et al. Structurally modified curcumin analogs inhibit STAT3 phosphorylation and promote apoptosis of human renal cell carcinoma and melanoma cell lines. PLoS One. 2012; 7(8): 40724

23. Ikenouchi J, Furuse M, Furuse K, Sasaki H, Tsukita S, Tsukita S. Tricellulin constitutes a novel barrier at tricellular contacts of epithelial cells. J Cell Biol. 2005;171(6):939-945.
24. Suzuki T, Yoshinaga N, Tanabe S. Interleukin-6 (IL-6) regulates claudin-2 expression and tight junction permeability in intestinal epithelium. J Biol Chem. 2011;286(36):31263-31271.

25. Zhang C, Sherman MP, Prince LS, et al. Paneth cell ablation in the presence of Klebsiella pneumoniae induces necrotizing enterocolitis (NEC)-like injury in the small intestine of immature mice. Dis Model Mech. 2012;5(4):522-532.

26. Musemeche C, Caplan M, Hsueh W, Sun X, Kelly A. Experimental necrotizing enterocolitis: the role of polymorphonuclear neutrophils. J Pediatr Surg. 1991;26(9):1047-1049. discussion 1049-1050.

27. Cani PD, Bibiloni R, Knauf C, et al. Changes in gut microbiota control metabolic endotoxemia-induced inflammation in high-fat diet-induced obesity and diabetes in mice. Diabetes. 2008;57(6):1470-1481.

28. Gupta J, Nebreda AR. Analysis of intestinal permeability in mice. Bioprotocol. 2014;4(22):e1289.

29. Hackam DJ, Good M, Sodhi CP. Mechanisms of gut barrier failure in the pathogenesis of necrotizing enterocolitis: Toll-like receptors throw the switch. Semin Pediatr Surg. 2013;22(2):76-82.

30. Liu Y, Fatheree NY, Mangalat N, Rhoads JM. Lactobacillus reuteri strains reduce incidence and severity of experimental necrotizing enterocolitis via modulation of TLR4 and NF- $\mathrm{KB}$ signaling in the intestine. Am J Physiol Gastrointest Liver Physiol. 2012;302(6):G608-G617.

31. Halpern MD, Denning PW. The role of intestinal epithelial barrier function in the development of NEC. Tissue Barriers. 2015;3(1-2): e1000707.

32. Claud EC, Walker WA. Bacterial colonization, probiotics, and necrotizing enterocolitis. J Clin Gastroenterol. 2008;42(suppl 2):S46-S52.

33. Hull MA, Fisher JG, Gutierrez IM, et al. Mortality and management of surgical necrotizing enterocolitis in very low birth weight neonates: a prospective cohort study. J Am Coll Surg. 2014;218(6):1148-1155.

34. Jilling T, Simon D, Lu J, et al. The roles of bacteria and TLR4 in rat and murine models of necrotizing enterocolitis. J Immunol. 2006; 177(5):3273-3282.

35. Lu P, Sodhi CP, Hackam DJ. Toll-like receptor regulation of intestinal development and inflammation in the pathogenesis of necrotizing enterocolitis. Pathophysiology. 2014;21(1):81-93.

36. Claud EC, Walker WA. Hypothesis: inappropriate colonization of the premature intestine can cause neonatal necrotizing enterocolitis. FASEB J. 2001;15(8):1398-1403.

37. Lin PW, Nasr TR, Stoll BJ. Necrotizing enterocolitis: recent scientific advances in pathophysiology and prevention. Semin Perinatol. 2008;32(2):70-82.

38. Bill MA, Fuchs JR, Li C, et al. The small molecule curcumin analog FLLL32 induces apoptosis in melanoma cells via STAT3 inhibition and retains the cellular response to cytokines with anti-tumor activity. Mol Cancer. 2010;9:165.

39. Fossey SL, Bear MD, Lin J, et al. The novel curcumin analog FLLL32 decreases STAT3 DNA binding activity and expression, and induces apoptosis in osteosarcoma cell lines. BMC Cancer. 2011;11:112.

40. Soliman A, Michelsen KS, Karahashi H, et al. Platelet-activating factor induces TLR4 expression in intestinal epithelial cells: implication for the pathogenesis of necrotizing enterocolitis. PLoS One. 2010;5(10):e15044.

41. Eckert J, Scott B, Lawrence SM, et al. FLLL32, a curcumin analog, ameliorates intestinal injury in necrotizing enterocolitis. Poster presented at: Pediatric Academic Society Meeting, April 28, 2015; San Diego, CA, USA. 
The Journal of Inflammation Research is an international, peer-reviewed open access journal that welcomes laboratory and clinical findings on the molecular basis, cell biology and pharmacology of inflammation including original research, reviews, symposium reports, hypothesis formation and commentaries on: acute/chronic inflammation; mediators of

Submit your manuscript here: https://www.dovepress.com/journal-of-inflammation-research-journa

inflammation; cellular processes; molecular mechanisms; pharmacology and novel anti-inflammatory drugs; clinical conditions involving inflammation. The manuscript management system is completely online and includes a very quick and fair peer-review system. Visit http://www.dove press.com/testimonials.php to read real quotes from published authors. 